\title{
Narrow-Beam Argon Ion Milling of Carbon-Supported Ex Situ Lift-Out FIB Specimens
}

\author{
M.J. Campin ${ }^{1}$, C.S. Bonifacio ${ }^{1}$, H.H. Kang ${ }^{2}$, P. Nowakowski ${ }^{1}$, M. Boccabella ${ }^{1}$, and P.E. Fischione ${ }^{1}$ \\ 1. E.A. Fischione Instruments, Inc., Export, PA,USA \\ 2. Center for Complex Analysis, GLOBALFOUNDRIES, Malta, NY, USA
}

Focused ion beam (FIB) tools are used to prepare transmission electron microscopy (TEM) specimens due to the site specificity and accuracy of specimen thinning and extraction that it provides $[1,2]$. The preparation of TEM specimens using gallium-based FIB tools with in situ lift-out capability has been the standard for characterization and failure analysis of materials and devices in the semiconductor industry for many years [3]. To further improve throughput in the semiconductor manufacturing industry, an inline TEM sample preparation method with ex situ lift-out (EXLO) technique on a perforated, carbon supported, mesh-type TEM grid was introduced [3, 4]. Unfortunately, the carbon support presents problems if the lamella needs to be thinned for high-resolution TEM analysis or if one is attempting to precisely determine the region of interest on the device. Further thinning of the specimen on a carbon support is limited due to preferential milling of the carbon support, which could result in loss of the specimen. In this work, we introduce the feasibility of using the narrow-beam argon ion milling capabilities of the PicoMill TEM specimen preparation system [Fischione Instruments] to improve carbon-supported EXLO specimens without compromising the integrity of the support and to end-point on specific features. The result is electron transparent and gallium-free specimens that contain only the features of interest.

TEM lamellae from a semiconductor wafer were prepared using a CLM dual beam FIB [FEI]. In this work, we intentionally produced thick specimens with expected thickness of $100 \mathrm{~nm}$ or more from the area of interest. Tests to determine the optimum ion milling conditions (e.g., milling rates and current densities) were performed (Figure 1a), after which the specimens were ion milled on each side at decreasing ion energies (Figure 1b). TEM and energy-dispersive X-ray spectroscopy (EDS) characterization using a Tecnai F30 [FEI] operated at $300 \mathrm{kV}$ were performed before and after milling.

Qualitative thickness reduction was determined in situ by observing the change in brightness and contrast of the images from both the secondary electron detector (SED) and scanning transmission electron microscope (STEM) detector in the PicoMill system (Figure 1b). The low current densities of the ion beam allowed thinning of the lamella with minimal damage to the supporting carbon foil. In addition, the sub-micron beam size and precise ion beam positioning possible with the PicoMill system allowed thinning of both sides of the lamella within the $5 \mu \mathrm{m}$ opening of the carbon support. EDS spectra acquired before and after milling of the specimen show a significant reduction of Ga (Figure 2). Quantitative analysis of the thickness and Ga reduction is in progress.

References:

[1] LA Giannuzzi and FA Stevie, Micron 30 (1999), p. 197.

[2] J Mayer et al, MRS Bull. 30 (2007), p. 400.

[3] U Muehle et al in "Microscopy: Science, technology, applications and education", eds. A MéndezVilas and J Díaz (FORMATEX, Badajoz), p. 1704. 
[4] HH Kang, et al, Conf. Proc. Int. Symp. Test. Failure Anal. (2010), p. 102.
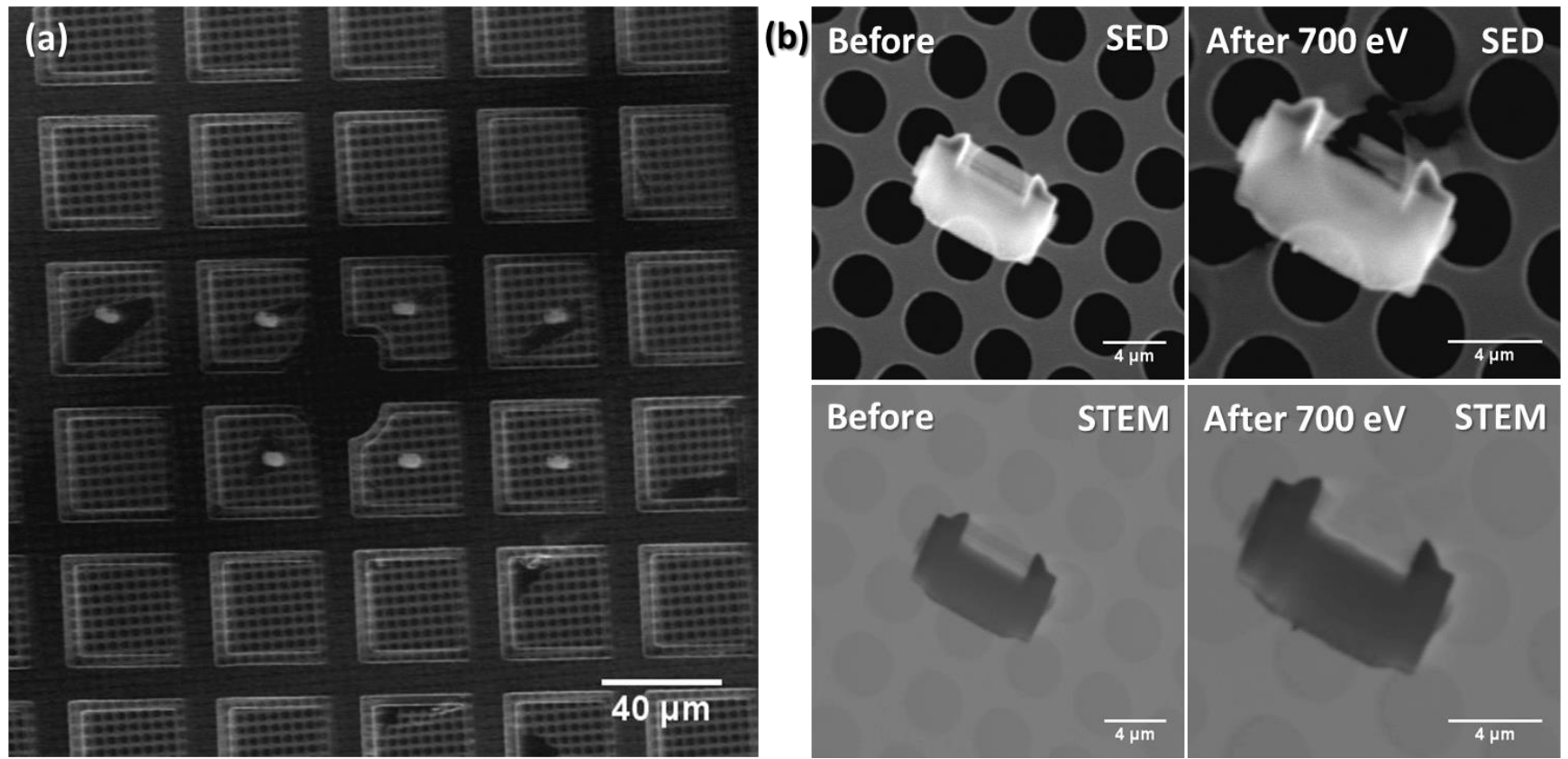

Figure 1. A SED image (a) from the PicoMill system after testing the optimum conditions for milling the carbon-supported, ex situ lift-out specimens. SED and STEM PicoMill system images (b) before and after milling showing an intact TEM lamella after milling at $700 \mathrm{eV}$.
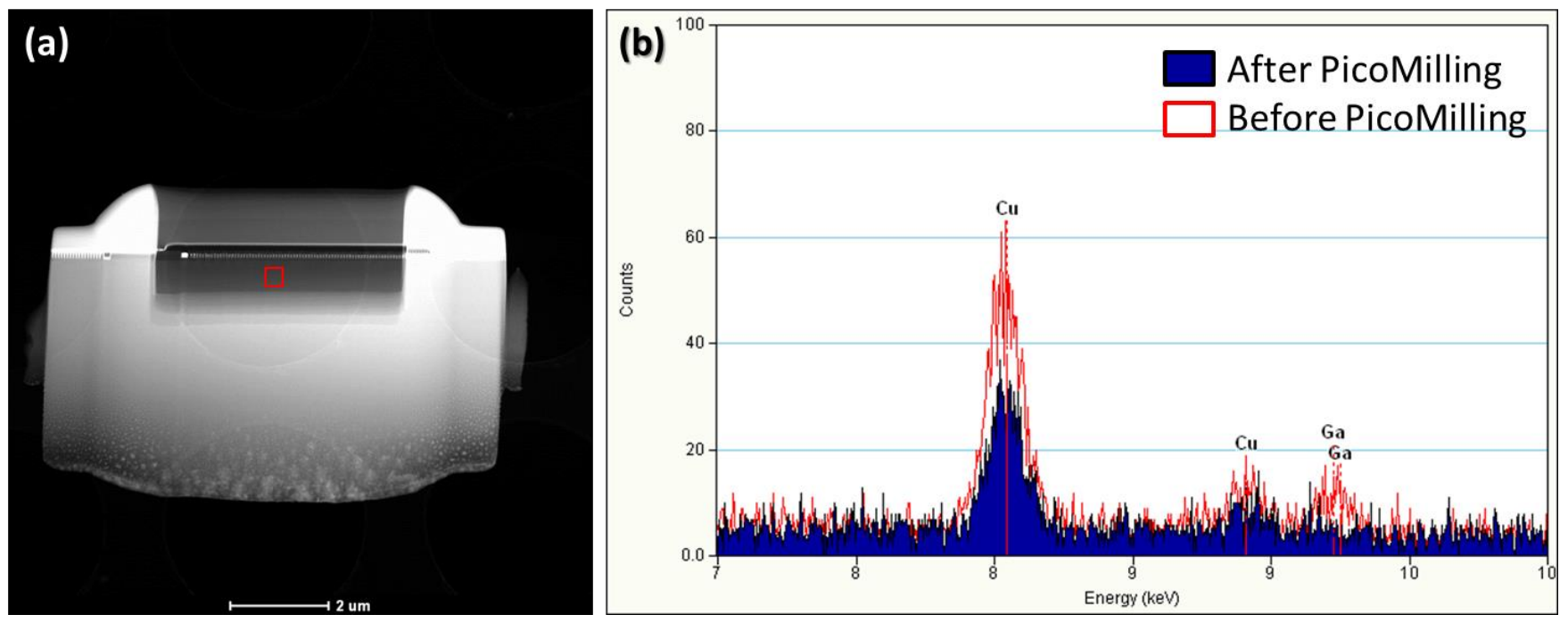

Figure 2. High angle annular dark field STEM image (a) of the carbon-supported, ex situ lift-out lamella. The red square indicates the area of EDS acquisition. EDS data (b) acquired before milling (red) and after milling (blue) show a significant reduction in the amount of gallium. The carbon is from the mesh grid supporting the carbon foil. 